\title{
EFEITOS DA PRÁTICA REGULAR DE EXERCÍCIOS FÍSICOS POR PORTADORES DE LÚPUS ERITEMATOSO SISTÊMICO: ESTUDO DE REVISÃO
}

Cátia Fernandes Leite, Universidade Federal de Pelotas - UFPEL, Pelotas, Rio Grande do Sul-Brasil

Marcio Neres dos Santos, Universidade Federal de Pelotas - UFPEL, Pelotas, Rio Grande do Sul - Brasil

Airton José Rombaldi, Universidade Federal de Pelotas - UFPEL, Pelotas, Rio Grande do Sul-Brasil

\section{RESUMO}

A quantidade de estudos relacionando os efeitos do exercício físico sobre parâmetros imunológicos e fisiológicos em pacientes com lúpus eritematoso sistêmico (LES) ainda é restrita. Objetivo: Revisar a literária a respeito dos efeitos do exercício sobre parâmetros imunológicos e fisiológicos em pacientes com LES. Procedimentos Metodológicos: Foi realizada uma busca nas bases de dados PubMed e Scielo e no portal de periódicos Capes. Os estudos foram selecionados pela leitura e análise de títulos, resumos e artigos completos. Resultados: No total foram encontrados 140 estudos relacionados ao tema. Após adoção dos critérios de exclusão, seis estudos fizeram parte da análise crítica do conteúdo deste estudo, dentre os quais dois demonstraram haver alterações imunológicas e quatro melhorias nos parâmetros fisiológicos com a execução de exercícios físicos em pacientes com LES. Conclusão: O exercício físico parece ocasionar melhorias na capacidade física, funcional e na função imunológica de indivíduos com LES em baixa atividade da doença. Todavia, existem ainda muitos aspectos que devem ser esclarecidos em relação aos componentes imunológicos e fisiológicos de indivíduos com LES submetidos a programas de exercícios físicos.

Palavras-Chave: Lúpus Eritematoso Sistêmico; Exercício; Imunidade; Aptidão física.

\section{EFFECTS OF THE REGULAR PRATICE OF PHYSICAL EXERCISES BY PATIENTS WITH SYSTEMIC LUPUS ERYTHEMATOSUS: A REVISION STUDY}

\begin{abstract}
The number of studies relating the effects of physical exercise on physiological and immunological parameters in patients with systemic lupus erythematosus (LES) is still restricted. Objective: To review the literature concerning the effects of exercise on physiological and immunological parameters in patients with LES. Methodological Procedures: We performed a search in the databases of PubMed and Scielo and portal Capes. The studies were selected by reading and analysis of titles, abstracts and full papers. Results: In total, 140 studies were found related to the topic. After adoption of the exclusion criteria, six studies were part of the review of the content of this study, two of
\end{abstract}


which have demonstrated immunological changes and improvements in the four physiological parameters by performing exercise in patients with LES. Conclusion: Exercise seems to bring about improvements in physical capacity, functional and immune function of patients with LES in low disease activity. However, there are still many aspects that must be clarified in relation to immunological and physiological components of individuals with LES undergoing physical exercise programs.

Key-Words: Lupus Erythematosus Systemic; Exercise; Immunity; Physical fitness.

\section{EFECTOS DE LA PRÁCTICA REGULAR DE EJERCICIO FÍSICO EN PACIENTES CON LUPUS ERITEMATOSO SISTÉMICO: ESTUDIO DE REVISIÓN}

\section{RESUMEN}

El número de estudios sobre los efectos del ejercicio físico sobre parámetros fisiológicos e inmunológicos en pacientes con lupus eritematoso sistémico (LES) es todavía limitada. Objetivo: Revisar la literatura sobre los efectos del ejercicio sobre parámetros fisiológicos e inmunológicos en pacientes con LES. Procedimiento metodológico: Se realizó una búsqueda en las bases de datos de revistas en PubMed y Scielo y portal de búsqueda Capes. Los estudios fueron seleccionados mediante la lectura y análisis de los títulos, resúmenes y trabajos completos. Resultados: En total, se encontraron 140 estudios relacionados con el tema. Después de la adopción de los criterios de exclusión, seis estudios fueron incluidos en la revisión del contenido para este estudio, dos de los cuales han demostrado alteraciones inmunológicas y las mejoras en los cuatro parámetros fisiológicos mediante la realización de ejercicio en pacientes con LES. Conclusión: El ejercicio parece producir mejoras en la capacidad física, funcional y la función inmune de los pacientes con LES en la actividad de la enfermedad. Sin embargo, hay muchos aspectos que hay que aclarar en relación a los componentes inmunológicos y fisiológicos de los individuos con LES sometidos a programas de ejercicio físico.

Palabras-Clave: Lupus Eritematoso Sistémico; Ejercicio; Inmunidad; Acondicionamiento físico. 


\section{INTRODUÇÃO}

O lúpus eritematoso sistêmico (LES) é uma doença auto-imune, crônica, inflamatória, de etiologia desconhecida e que afeta múltiplos órgãos com sintomas localizados ou sistêmicos. ${ }^{1}$ Esta desordem auto-imune na qual o sistema imunológico do corpo incorretamente ataca seus próprios tecidos e órgãos conduzindo a inflamação e danos dos mesmos, em geral afeta mulheres em idade reprodutiva, mas também ocorre em crianças, adolescentes e homens. ${ }^{2}$ A prevalência desta enfermidade na população geral oscila de 15 a $50 / 100.000$ habitantes. $^{3}$

Doenças auto-imunes, tais como o LES, são causadas pelo desequilíbrio imunológico e pela perda da auto-tolerância. ${ }^{4} \mathrm{O}$ LES é uma doença secundária a alterações no funcionamento dos linfócitos B e linfócitos T. ${ }^{5}$ Este distúrbio imunológico pode ser devido a uma ativação de células B policlonais que induz a produção em grande número de autoanticorpos. ${ }^{6}$ Estes auto-anticorpos e o complexo imune acumulam-se nas articulações, nos rins e em outros tecidos provocando respostas inflamatórias e conduzindo a danos e destruições de tecidos e órgãos. ${ }^{7}$

O LES ataca principalmente a pele, os músculos, as articulações, os rins, o coração, os vasos sanguíneos de pequeno e médio calibre, o sangue, os pulmões e o sistema nervoso central, com evolução de exacerbações e remissões alternadas e manifestações polimórficas. ${ }^{8}$ A desordem pode ser leve em alguns casos, como por exemplo, envolvendo somente a pele, e em outros casos, muito grave, afetando múltiplos órgãos. O curso da doença é caracterizado por intervalos da doença ativa e remissões (intervalos da doença inativa). ${ }^{2}$ As manifestações gerais do LES são fadiga, febre, perda de peso corporal e linfoadenopatia. E as manifestações específicas ocorrem conforme os comprometimentos localizados em diversos órgãos ou sistemas. ${ }^{1}$

Manter a qualidade de vida dos portadores de LES é de extrema importância o que requer uma ampla discussão sobre os principais fatores associados a este tema, como por exemplo, a prática regular de exercícios físicos. Entretanto, a quantidade de estudos relacionando os efeitos do treinamento físico sobre parâmetros imunológicos e fisiológicos em pacientes com LES ainda é restrita, sendo necessário o desenvolvimento de maiores 
investigações a respeito desse tema. Nesse sentido, o objetivo deste estudo foi realizar uma revisão da literatura disponível a respeito dos efeitos do exercício físico sobre as concentrações celulares do sistema imunológico e sobre parâmetros fisiológicos de pacientes portadores de LES.

\section{PROCEDIMENTOS METODOLÓGICOS}

Foi realizada uma busca nas bases de dados PubMed e Scielo, e nos portais de periódicos Capes e Portal da Pesquisa utilizando os seguintes descritores: systemic erythematosus lupus and immune system (or immunity) and physical activity (or physical exercise). A busca pelos artigos nas bases de dados e nos portais foi realizada nos meses de fevereiro a agosto de 2010. Os estudos foram selecionados pela leitura e análise de títulos, resumos e artigos completos. Aqueles trabalhos que apresentaram no título combinações envolvendo algum dos descritores foram inicialmente pré-selecionados para análise.

Os critérios definidos a priori para seleção dos estudos foram: 1) amostras constituídas por portadores de LES; 2) avaliação dos efeitos agudos ou crônicos dos exercícios físicos em pacientes com lúpus; 3) verificação dos efeitos do exercício físico sobre componentes imunológicos e fisiológicos em pessoas com LES.

Também foram adotados os seguintes critérios de exclusão: 1) amostras compostas por portadores de patologias associadas ao LES; 2) investigação do uso de medicamentos a pacientes com LES, porém sem avaliar o efeito agudo ou crônico do exercício físico nesta população; e 3) estudos experimentais com modelos animais. Desse modo, foram selecionados seis trabalhos para a análise crítica do conteúdo.

\section{RESULTADOS E DISCUSSÃO}

Após a exclusão das publicações duplicadas, o processo de busca retornou um total de 140 estudos relacionados ao tema. Destes, cinco foram excluídos pela análise de títulos e 93 pelo resumo, totalizando 42 artigos para leitura na íntegra. Os 93 artigos excluídos pelo resumo não atenderam aos critérios de inclusão definidos a priori por este estudo. Logo após, a análise na íntegra dos 42 artigos restantes, 36 foram excluídos. Seis estudos encontraram associações entre os efeitos do exercício físico, células imunológicas e Conexões: revista da Faculdade de Educação Física da UNICAMP, Campinas, v. 11, n. 3, p. 166-175, jul./set. 2013. ISSN: 1983-9030 
aspectos fisiológicos em pacientes com LES. Dentre estes, dois demonstraram haver alterações nas concentrações de células imunes e quatro melhorias nos parâmetros fisiológicos com a execução de exercícios físicos em pacientes com LES. O Quadro 1 resume os principais efeitos observados sobre estes parâmetros em portadores de LES. 
Quadro1 - Resumo dos estudos sobre os efeitos dos exercícios físicos em pacientes com LES

\begin{tabular}{|c|c|c|c|c|c|c|}
\hline Ano & 1981 & 2003 & 2004 & 2005 & 2005 & 2008 \\
\hline AUTOR & $\begin{array}{l}\text { ISENBERG et } \\
\text { al. }\end{array}$ & $\begin{array}{l}\text { KEYSER et } \\
\text { al. }\end{array}$ & POOL et al. & $\begin{array}{l}\text { CARVALHO } \\
\text { et al. }\end{array}$ & $\begin{array}{l}\text { CLARKE- } \\
\text { JENSSEN et } \\
\text { al. }\end{array}$ & $\begin{array}{l}\text { HOUGHTON } \\
\text { et al. }\end{array}$ \\
\hline AMOSTRA & $\begin{array}{l}\text { Mulheres com } \\
\text { média de idade } \\
\text { de } 28 \text { anos } \\
\text { participaram do } \\
\text { estudo realizado } \\
\text { na University } \\
\text { College } \\
\text { Hospital, } \\
\text { London }\end{array}$ & $\begin{array}{l}\text { Mulheres entre } \\
26 \text { e } 44 \text { anos de } \\
\text { idade e com } \\
\text { baixa atividade } \\
\text { da doença, } \\
\text { protocolo } \\
\text { aprovado pela } \\
\text { University of } \\
\text { Maryland, } \\
\text { Baltimore }\end{array}$ & $\begin{array}{l}\text { Pacientes com } \\
\text { LES (média de } \\
\text { idade } 33 \text { anos) } \\
\text { e pacientes com } \\
\text { artrite } \\
\text { reumática } \\
\text { (média de idade } \\
35 \text { anos) } \\
\text { selecionados da } \\
\text { clínica de } \\
\text { reumatologia } \\
\text { em St George's } \\
\text { Hospital, } \\
\text { London }\end{array}$ & $\begin{array}{l}\text { Mulheres entre } \\
18 \text { e } 55 \text { anos de } \\
\text { idade, } \\
\text { acompanhadas } \\
\text { pela divisão de } \\
\text { reumatologia } \\
\text { dos hospitais } \\
\text { universitários } \\
\text { da Universidade } \\
\text { Federal de São } \\
\text { Paulo e da } \\
\text { Santa Casa de } \\
\text { Misericórdia de } \\
\text { São Paulo }\end{array}$ & $\begin{array}{l}\text { Mulheres acima } \\
\text { dos } 18 \text { anos de } \\
\text { idade, } \\
\text { recrutadas de } \\
\text { uma clínica em } \\
\text { Rikshospitalet } \\
\text { University } \\
\text { Hospital, Oslo, } \\
\text { Norway e sem } \\
\text { doença ativa } \\
\text { em nenhum } \\
\text { órgão vital }\end{array}$ & $\begin{array}{l}\text { Pacientes } \\
\text { jovens, idade } \\
\text { entre } 12 \text { a } 20 \\
\text { anos, } \\
\text { acompanhados } \\
\text { pelo Pediatric } \\
\text { Rheumatology } \\
\text { Program at } \\
\text { British } \\
\text { Columbia } \\
\text { Children's } \\
\text { Hospital } \\
\text { participaram do } \\
\text { estudo }\end{array}$ \\
\hline $\begin{array}{c}\text { PROGRAMAS } \\
\text { DE EXERCICIO } \\
\text { FISICO }\end{array}$ & $\begin{array}{l}\text { Exercício } \\
\text { aeróbio agudo }\end{array}$ & $\begin{array}{l}\text { Teste para } \\
\text { determinar o } \\
\text { consumo de } \\
\text { oxigênio de } \\
\text { pico }\left(\mathrm{VO}_{2 \text { pico }}\right)\end{array}$ & $\begin{array}{l}\text { Exercício } \\
\text { aeróbio agudo } \\
\text { realizado em } \\
\text { ciclo ergômetro }\end{array}$ & $\begin{array}{lr}\text { Programa de } \\
\text { caminhada com } \\
\text { duração de } & 12 \\
\text { semanas, } & \\
\text { realizada } & 3 \\
\text { vezes } & \text { por } \\
\text { semana e } & 60 \\
\text { min por sessão }\end{array}$ & $\begin{array}{lr}\text { Programa } & \text { de } \\
\text { caminhada, } & 3 \\
\text { vezes } & \text { por } \\
\text { semana } & \mathrm{e} \\
\text { durante } & 12 \\
\text { semanas } & \end{array}$ & $\begin{array}{l}\text { Testes para } \\
\text { determinações } \\
\text { do } \mathrm{VO}_{2 \text { pico }} \text { e da } \\
\text { capacidade } \\
\text { aeróbia } \\
\text { submáxima }\end{array}$ \\
\hline $\begin{array}{l}\text { PRINCIPAIS } \\
\text { VARIÁVEIS }\end{array}$ & $\begin{array}{l}\text { Níveis } \\
\text { circulantes de } \\
\text { complexo imune } \\
(\text { conteúdo de } \\
\left.\text { IgG - } \mu \mathrm{g} \cdot \mathrm{mL}^{-1}\right)\end{array}$ & $\begin{array}{l}\mathrm{VO}_{2 \text { pico, }} \\
\text { quantificação } \\
\text { da severidade } \\
\text { da fadiga }\end{array}$ & $\begin{array}{l}\text { Concentrações } \\
\text { de cortisol, } \\
\text { prolactina e } \\
\text { linfócitos CD4+ } \\
\text { e CD8+ }\end{array}$ & $\begin{array}{l}\text { Capacidade } \\
\text { aeróbia, fadiga, } \\
\text { pulso de } \\
\text { oxigênio, limiar } \\
\text { anaeróbio } \\
\text { ventilatório }\end{array}$ & $\begin{array}{c}\text { Capacidade } \\
\text { aeróbia } \\
\left(\mathrm{VO}_{2} \mathrm{max}\right) \\
\text { percepção de } \\
\text { dor e de fadiga }\end{array}$ & $\begin{array}{l}\mathrm{VO}_{2 \text { pico, }} \text { peso } \\
\text { corporal, } \\
\text { estatura, índice } \\
\text { de massa } \\
\text { corporal, } \\
\text { quantificação } \\
\text { da severidade } \\
\text { da fadiga }\end{array}$ \\
\hline RESULTADOS & $\begin{array}{l}\text { Houve queda } \\
\text { nos níveis } \\
\text { circulantes do } \\
\text { complexo imune } \\
\text { após o exercício } \\
\text { físico }\end{array}$ & $\begin{array}{l}\text { Houve } \\
\text { indicação que a } \\
\text { severidade da } \\
\text { fadiga limitou a } \\
\text { atividade física } \\
\text { das pacientes } \\
\text { com LES }\end{array}$ & $\begin{array}{l}\text { Reduções nos } \\
\text { níveis de } \\
\text { cortisol. } \\
\text { Aumento não } \\
\text { significativo } \\
\text { nos níveis de } \\
\text { prolactina. } \\
\text { Reduções } \\
\text { significativas } \\
\text { nas } \\
\text { concentrações } \\
\text { de células T } \\
\text { CD4+ no pico } \\
\text { do exercício e } \\
\text { com aumento } \\
\text { nos níveis no } \\
\text { pós-exercício. } \\
\text { Reduções } \\
\text { significativas } \\
\text { no pós- } \\
\text { exercício nas } \\
\text { concentrações } \\
\text { de células T } \\
\text { CD8+ }\end{array}$ & 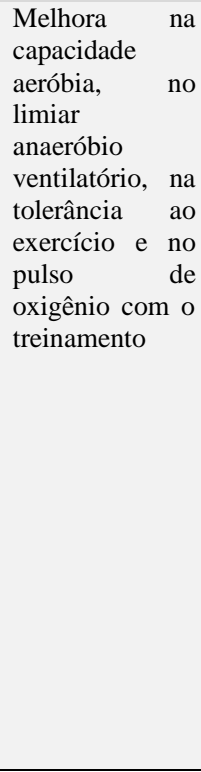 & $\begin{array}{l}\text { Melhora no } \\
\mathrm{VO}_{2} \text { max. } \\
\text { Nenhum } \\
\text { agravamento } \\
\text { relacionado à } \\
\text { dor, fadiga ou } \\
\text { função física } \\
\text { durante e após } \\
\text { o treinamento }\end{array}$ & $\begin{array}{l}\text { Não houve } \\
\text { correlação } \\
\text { significativa } \\
\text { entre fadiga e } \\
\text { as medidas de } \\
\text { aptidão física. } \\
\text { Nem a aptidão } \\
\text { física e nem a } \\
\text { fadiga } \\
\text { apresentaram } \\
\text { correlação } \\
\text { significativa } \\
\text { com a atividade } \\
\text { da doença e os } \\
\text { possíveis danos } \\
\text { proporcionados } \\
\text { pela doença }\end{array}$ \\
\hline
\end{tabular}

A realização de exercícios físicos por pacientes com LES poderá ocasionar melhorias em alguns parâmetros fisiológicos. O treinamento cardiovascular supervisionado é bem tolerado por pacientes com LES e melhora de forma significativa a tolerância individual ao 
exercício, a capacidade aeróbia, o pulso de oxigênio, a fadiga, a qualidade de vida, a depressão e a capacidade funcional destes indivíduos. ${ }^{9} \mathrm{O}$ exercício aeróbio não agrava a atividade da doença em qualquer momento durante as seções de exercícios e pode ter efeitos benéficos sobre a função física de pacientes com LES com baixa atividade da doença. ${ }^{10}$ Além disso, não há correlação entre a aptidão física e a fadiga em relação a atividade da doença. ${ }^{11}$

A melhoria da qualidade de vida em portadores de LES acompanhada por benefícios físicos e funcionais decorrentes do exercício físico foram verificados por alguns estudos. Uma pesquisa observou que em mulheres com LES entre 18 e 55 anos de idade, acompanhadas pela divisão de reumatologia dos hospitais universitários da Universidade Federal de São Paulo e da Santa Casa de Misericórdia de São Paulo, submetidas a um programa de caminhada, realizado três vezes por semana com duração de 12 semanas, resultou em melhoria na capacidade aeróbia, no limiar anaeróbio ventilatório, na tolerância ao exercício e no pulso de oxigênio. ${ }^{9}$ Outro estudo identificou que em mulheres acima dos 18 anos de idade, portadoras de LES, recrutadas de uma clínica em Rikshospitalet University Hospital, Oslo, Noruega, e sem doença ativa nos órgãos vitais durante o período de treinamento, houve melhora no $\mathrm{VO}_{2} \max$ (consumo máximo de oxigênio) e nenhum agravamento relacionado à dor, fadiga ou função física durante e após um programa de caminhada em esteira rolante a $70 \%$ da FCmax (frequência cardíaca máxima), realizado três vezes por semana e durante 12 semanas. ${ }^{10}$ Entretanto, outros pesquisadores ao aplicarem um teste para determinar o $\mathrm{VO}_{2 \text { pico }}$ de mulheres com LES, em idades entre $26 \mathrm{e}$ 44 anos e com baixa atividade da doença, observaram que a severidade da fadiga limitou a atividade física destas pacientes. ${ }^{12}$

Alguns parâmetros imunológicos podem sofrer variações em suas concentrações com a execução de exercícios físicos em pacientes com LES. Ao analisar o efeito do exercício aeróbio agudo realizado em cicloergômetro por mulheres com LES, média de idade de 33 anos, e mulheres com artrite reumática, média de idade 35 anos, selecionadas da clínica de reumatologia em St George's Hospital, London, observaram-se reduções significativas nas concentrações de células $\mathrm{T} \mathrm{CD}^{+}$no pico do exercício e com aumento destes níveis no pósexercício. ${ }^{13}$ Além disso, reduções significativas no pós-exercício nas concentrações de 
células $\mathrm{T} \mathrm{CD} 8+{ }^{+13}$ Em outro estudo realizado em mulheres com média de idade de 28 anos portadoras de LES e submetidas a exercício aeróbio agudo desenvolvido em bicicleta ergométrica, observaram-se que os níveis circulantes do complexo imune (conteúdo de IgG - $\mu \mathrm{g} . \mathrm{mL}^{-1}$ ) diminuíram após o exercício físico. ${ }^{14}$

Apesar das melhorias decorrentes do exercício físico sobre alguns parâmetros imunes e fisiológicos em pacientes com LES apresentados pelo presente estudo, alguns fatores limitantes devem ser considerados em decorrência de aspectos metodológicos dos estudos analisados. Além das complicações ocasionadas pela LES previamente citadas devem-se ser levadas em consideração as doenças reumáticas associadas, como a artrite, as dores articulares e musculares e a fadiga. Além disso, devem-se considerar as próprias limitações do paciente quanto à capacidade funcional e a fase ativa da doença. Outro fator complicador é a perda da capacidade cardiocirculatória e cardiopulmonar do paciente o que pode comprometer a capacidade física e funcional destes indivíduos. Entretanto, os estudos demonstraram que os exercícios aeróbios são bem tolerados por esta população. Outra limitação apresentada por alguns dos estudos foi o pequeno número de pacientes participantes dos estudos o que inviabiliza a extrapolação dos dados para esta população.

Com base nos estudos aqui apresentados e discutidos, conclui-se que os exercícios físicos parecem ocasionar melhorias na capacidade física e funcional de indivíduos com LES. O engajamento desses indivíduos em rotinas de exercícios físicos pode ser benéfico, pois possibilita melhorias na função imunológica destes indivíduos com baixa atividade da doença. Todavia, existem ainda muitos aspectos que devem ser esclarecidos em relação aos componentes imunológicos e fisiológicos de indivíduos com LES, sugerindo assim o desenvolvimento maiores investigações nessa área.

\section{REFERÊNCIAS}

${ }^{1}$ REIS, M. G.; LOUREIRO, M. D. R.; SILVA, M. G. Aplicação da metodologia da assistência a pacientes com Lúpus Eritematoso Sistêmico em pulsoterapia: uma experiência docente. Revista Brasileira de Enfermagem, Brasília, v. 60, n. 2, p. 229-232, 2007.

Conexões: revista da Faculdade de Educação Física da UNICAMP, Campinas, v. 11, n. 3, p. 166-175, jul./set. 2013. ISSN: 1983-9030 
${ }^{2}$ RINGOLD, S.; LYNM, C.; GLASS, R. M. Systemic lupus erythematosus. JAMA: the journal of the American Medical Association, v. 293, n. 24, p. 3130, 2005.

${ }^{3}$ TELLES, R. W. et al. Frequência de doença cardiovascular aterosclerótica e de seus fatores de risco em pacientes com lúpus eritematoso sistêmico. Revista Brasileira de Reumatologia, São Paulo, v. 47, n. 3, p. 165-173, 2007.

${ }^{4}$ ROSA, S. B. et al. The use of stem cells for the treatment of autoimmune diseases. Brazilian Journal of Medical and Biological Research, Ribeirão Preto, v. 40, n. 12, p. 1579-1597, 2007.

${ }^{5}$ TISKIEVICZ, F. et al. Prolactina e macroprolactina no lúpus eritematoso sistêmico (LES). Revista Brasileira de Reumatologia, São Paulo, v. 45, n. 3, p. 191-194, 2005.

${ }^{6}$ GARCIA, S.; CAMPO-DE-CARVALHO, A. C. Neonatal lupus syndrome: the heart as a target of the immune system. Anais da Academia Brasileira de Ciências, Rio de Janeiro, v. 72, n. 1, p. 83-90, 2000.

${ }^{7}$ MARSHAK-ROTHSTEIN, A.; RIFKIN, I. R. Immunologically active autoantigens: the role of toll-like receptors in the development of chronic inflammatory disease. Annual Review of Immunology, v. 25, p. 419-441, 2007.

${ }^{8}$ PERES, J. M. C.; TEDDE, P. F. B.; LAMARI, N. M. Fadiga nos portadores de lúpus eritematoso sistemático sob intervenção fisioterapêutica. O Mundo da Saúde, São Paulo, v. 30, n. 1, p. 141-145, 2006.

${ }^{9}$ CARVALHO, M. R. P. et al. Effects of supervised cardiovascular training program on exercise tolerance, aerobic capacity, and quality of life in patients with systemic lupus erythematosus. Arthritis and Rheumatism, v. 53, n. 6, p. 838-844, 2005.

Conexões: revista da Faculdade de Educação Física da UNICAMP, Campinas, v. 11, n. 3, p. 166-175, jul./set. 2013. ISSN: 1983-9030 
${ }^{10}$ CLARKE-JENSSEN, A. C. et al. Effects of supervised aerobic exercise in patients with systemic lupus erythematosus: a pilot study. Arthritis and Rheumatism, v. 53, n. 2, p. 308-312, 2005.

${ }^{11}$ HOUGHTON, K. M. et al. Fitness, fatigue, disease activity, and quality of life in pediatric lupus. Arthritis and Rheumatism, v. 59, n. 4, p. 537-545, 2008.

${ }^{12}$ KEYSER, R. E. et al. Evidence for aerobic insufficiency in women with systemic lupus erythematosus. Arthritis and Rheumatism, v. 49, n. 1, p. 16-22, 2003.

${ }^{13}$ POOL, A. J. et al. Serum cortisol reduction and abnormal prolactin and CD4+/CD8+ Tcell response as a result of controlled exercise in patients with rheumatoid arthritis and systemic lupus erythematosus despite unaltered muscle energetic. Rheumatology, v. 43, n.1, p. 43-48, 2004.

${ }^{14}$ ISENBERG, D. A. et al. Variation in circulating immune complex levels with diet, exercise, and sleep: a comparison between normal controls and patients with systemic lupus erythematosus. Annals of the Rheumatic Disease, v. 40, p. 466-469, 1981.

Recebido em: 21 dez. 2012 Aceito em: 10 ago. 2013 Contato: Airton José Rombaldi rombaldi@ufpel.tche.br 